\title{
EchoGéo
}

\section{La croissance urbaine au Brésil : concentration dans les métropoles ou rééquilibrage du système urbain?}

\author{
Cathy Chatel, François Moriconi-Ebrard and Maria Encarnação Beltrão \\ Sposito
}

\section{(2) OpenEdition} Journals

Electronic version

URL: https://journals.openedition.org/echogeo/15001

DOI: 10.4000/echogeo.15001

ISSN: 1963-1197

Publisher

Pôle de recherche pour l'organisation et la diffusion de l'information géographique (CNRS UMR 8586)

Electronic reference

Cathy Chatel, François Moriconi-Ebrard and Maria Encarnação Beltrão Sposito, "La croissance urbaine au Brésil : concentration dans les métropoles ou rééquilibrage du système urbain ?", EchoGéo [Online], 41 | 2017, Online since 28 September 2017, connection on 31 July 2021. URL: http:// journals.openedition.org/echogeo/15001; DOI: https://doi.org/10.4000/echogeo.15001

This text was automatically generated on 31 July 2021.

EchoGéo est mis à disposition selon les termes de la licence Creative Commons Attribution - Pas d'Utilisation Commerciale - Pas de Modification 4.0 International (CC BY-NC-ND) 


\title{
La croissance urbaine au Brésil : concentration dans les métropoles ou rééquilibrage du système urbain?
}

\author{
Cathy Chatel, François Moriconi-Ebrard and Maria Encarnação Beltrão \\ Sposito
}

\section{AUTHOR'S NOTE}

Cet article est issu d'un projet de recherche financé par la FAPESP (Fundação de Amparo à Pesquisa do Estado de São Paulo) intitulé "BRASIpolis" (Cidades, aglomerações urbanas e população - Processo n²014/07800-5) et développé par Cathy Chatel, dans le cadre du projet thématique "Lógicas econômicas e práticas espaciais contemporâneas: cidades médias e consumo" dirigé par Maria Encarnação Beltrão Sposito, laboratoire "Grupo de Pesquisa Produção do Espaço e Redefinições Regionais" (GAsPERR), Universidade Estadual Paulista (UNESP) - Câmpus de Presidente Prudente. http://www.bv.fapesp.br/pt/auxilios/47675/logicas-economicas-e-praticas-espaciaiscontemporaneas-cidades-medias-e-consumo/

\section{Introduction}

1 Le Brésil possède deux des megacities de la planète au sens de la définition des NationsUnies $^{1}$ : São Paulo et Rio de Janeiro. C'est l'un des 5 pays du monde à se trouver dans ce cas, à l'égal des États-Unis, mais derrière la Chine (qui compte 5 megacities) et l'Inde (4 megacities), qui sont des pays 6 à 7 fois plus peuplés, et le Japon (3 megacities). Si la faible superficie disponible alliée à une forte densité démographique et à une orographie tourmentée, explique aisément la concentration urbaine dans l'archipel nippon, les dimensions des deux megacities brésiliennes sont particulièrement 
surprenantes dans un pays offrant autant de possibilités de localisation, de diversité de ressources sur l'ensemble d'un territoire 22 fois plus vaste que le Japon.

Sur le terrain, des dizaines de millions d'habitants expérimentent au quotidien les conséquences de ces formidables concentrations humaines. Leurs aspects positifs effervescence culturelle, niveau de développement et d'équipement, production de richesse - comme leurs aspects négatifs - engorgement de la circulation et des moyens de transports, pollution, insécurité, inégalités sociales, économiques et spatiales figurent parmi les thèmes les plus médiatisés. On peut alors réellement expérimenter le fait que l'urbanisation s'étale physiquement et cette expérience naît d'une immersion dans un milieu : celui de l'agglomération urbaine.

3 Avant toute chose, il convient de remettre ici un peu d'ordre de grandeur dans les «masses » absolues des chiffres. En effet, une agglomération de 20 millions d'habitants dont la population croîtrait annuellement au taux dérisoire de $0,1 \%$ par an, gagnerait tout de même plus de 20000 habitants par an. Soit autant qu'une ville secondaire de 200000 habitants, cent fois moins peuplée, qui augmenterait au rythme vertigineux de $10 \%$ par an². Au-delà d'un certain point, la masse démographique, à elle seule, suffit à assurer un avantage numérique absolu pour la croissance, même si cette dernière est inférieure, voire très inférieure en valeur relative. Ce n'est donc pas tant les valeurs relatives de la croissance qui alarment l'opinion et que perçoivent les habitants, qu'une question de "masse», donc de chiffres absolus. Pénétrant dans l'une des deux megacities brésiliennes depuis n'importe quel point cardinal, on ne peut également que constater l'intensité browniennne des échanges de marchandises et de personnes avec l'extérieur, et surtout la vitalité de la construction immobilière. À ce stade, ce n'est donc pas la qualité intrinsèquement urbaine du processus qui est en cause, mais un simple mécanisme démographique. En effet, quand bien même le solde migratoire serait nul ou négatif, l'accroissement naturel suffit à entretenir la concentration métropolitaine.

4 À partir de ces chiffres absolus, surgit une autre idée, présente dans la littérature, depuis au moins le XIX ${ }^{\mathrm{e}}$ siècle : la croissance métropolitaine ne s'effectuerait-elle pas au détriment du reste du territoire ? La réponse est en théorie si évidente, qu'elle confine à la tautologie. En effet, si les megacities n'avaient pas eu constamment un taux de croissance supérieur à toutes les autres moyennes - nationale, autres villes ou campagnes - elles ne seraient tout simplement pas devenues... des megacities.

Cependant, trois facteurs minimisent cette idée d'une croissance prépondérante des métropoles au détriment des autres villes et territoires du Brésil. D’abord, le rythme de la croissance urbaine s'essouffle parce que l'accroissement naturel diminue au Brésil. Aussi, certaines métropoles voient leur population croître moins vite que dans d'autres villes, notamment parce qu'avec la déconcentration de l'activité industrielle ainsi que la disjonction géographique entre les fonctions de commandement des entreprises et leurs activités productives, une partie des industries ont quitté les métropoles pour les villes grandes et moyennes. Enfin, dans les régions d'occupation plus récente, les grandes entreprises en forte expansion n'ont pas besoin des petites villes ni même des métropoles voisines : un nouveau modèle d'urbanisation se développe au centre et au nord du pays, comme les cartes de cet article l'illustreront par la suite.

6 Pourtant, la métaphore de la prédation du territoire par les megacities demeure prégnante. Elle consolide la rhétorique qui consiste à personnifier l'organisme urbain, épaulant notamment certaines images, comme dévorer et consommer l'espace 
(Bruegmann, 2005). L'urbanisation détruirait un espace productif envisagé exclusivement sous sa fonction agricole nourricière, au profit d'un sol stérile et artificialisé qui deviendrait improductif. Or, on sait que le sol urbanisé n'est pas improductif: il abrite la production des biens manufacturés, des services indispensables à la population comme l'enseignement et la santé, et accessoirement des logements pour des millions d'êtres humains, souvent plus économes en surface que les campagnes, en raison de la fréquence d'un habitat élevé.

7 Dès lors, l'idée selon laquelle l'accumulation dans les grandes métropoles se poursuit peut être juste d'un point de vue absolu, tout en étant fausse d'un point de vue relatif. C'est cette question que nous proposons d'aborder dans cet article.

Prenant à contre-pied les classifications fonctionnelles du système urbain, cet article propose de partir de ce milieu, cette base expérimentable de ce qui constitue au fond l'environnement quotidien urbain: l'agglomération. La méthode utilisée consiste à remettre à plat dans sa totalité le peuplement du Brésil, pour redéfinir la hiérarchie urbaine par une approche bottom up. Elle s'oppose ainsi aux approches top-down qui définissent les villes par leurs fonctions et leur aire d'influence.

Illustration 1 - Vue de l'agglomération de São Paulo depuis le centre en direction de l'ouest

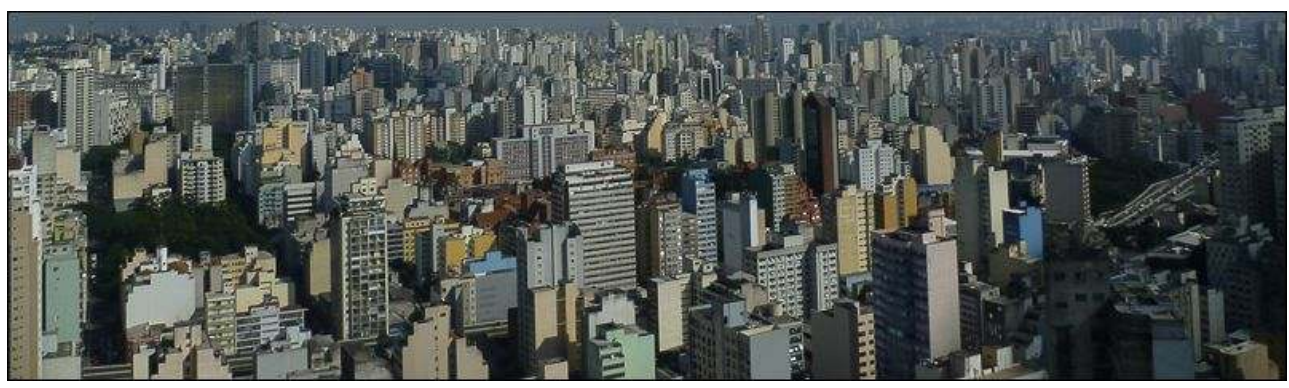

Auteur: F. Moriconi-Ebrard, 2015

\section{Définitions et méthodologie : l'espace urbain}

\section{Définition officielle de la population urbaine au Brésil}

Comme beaucoup d'auteurs, brésiliens en particulier, le reconnaissent, la définition nationale officielle de l'urbain est arbitraire : la population urbaine est la population comprise dans un périmètre défini légalement par les autorités locales du municipio, de la municipalité. Cette définition pose cependant plusieurs problèmes.

- Premièrement, la délimitation du périmètre urbain légal répond à des objectifs politiques de différents ordres: spéculation foncière, stratégie locale d'aménagement du territoire, intérêts particuliers, enjeux électoraux etc. Il en résulte que cette aire urbaine (área urbana) reprise par les statistiques de l'IBGE ${ }^{3}$ comprend en réalité des étendues non bâties plus ou moins vastes. Pour preuve, il existe des catégories de secteurs censitaires de l'aire urbaine légale appelées "aire urbaine non urbanisée [de cidade ou de vila]", Área urbana não urbanizada de cidade ou vila. Inversement, l'aire urbaine légale exclut parfois de facto des zones bâties de taille considérable, ce qui se traduit par un déficit de construction et d'entretien des infrastructures (égouts, électricité, poubelles...), ou de distribution des services (dispensaires locaux municipaux, transports en commun...). 
- Deuxièmement le siège administratif (sede) de tout municipio est automatiquement urbain, même s'il ne compte que quelques dizaines d'habitants. En 2016, 2448 municipios brésiliens comptent moins de 10000 habitants. Or il s'agit là de municipios entiers, et donc les 2448 villes comptent encore moins d'habitants.

- Enfin, comme les chiffres de la population urbaine sont agrégés par municipio par l'IBGE, ils laissent à penser qu'il y aurait 5572 aires urbaines légales au Brésil, soit 5572 villes. Or, cette idée est doublement fausse. Tout d'abord, la plupart des municipios sont divisés en distritos dont le siège a par définition son propre périmètre urbain, correspondant au cheflieu de distrito, la vila. Les cidades, correspondent aux sièges (sede) des municipios, et les vilas aux sièges des distritos. On obtient à première vue 10283 sites urbains physiquement géolocalisables, correspondant aux 10283 sièges de municipios et de distritos, et non pas seulement 5572 villes. Ensuite, la situation est encore plus complexe lorsqu'on découvre que chaque aire urbaine légale peut elle-même être constituée de différents blocs disjoints dans l'espace.

10 Si cette définition nationale est peut-être satisfaisante sur le plan institutionnel, elle n'est cependant pas utilisable dans une problématique de recherche fondée sur la morphologie, qui est ici au cœur de la question de la concentration urbaine. Dans ce cadre précis, la morphologie implique directement l'artificialisation du sol, la consommation d'espace, l'augmentation de la rente foncière. Ces caractères résultent de la combinaison entre masse démographique et continuum de constructions, et non pas de caractères sociologiques concernant l'urbanité de la population, ou de caractères politiques liés à la délimitation de périmètres administratifs.

11 C'est la raison pour laquelle, on utilise ici une méthodologie inédite au Brésil, qui consiste à réévaluer la population urbaine à partir d'une approche strictement morphologique. Le résultat de ce travail est la production d'une base de données appelée Brasipolis, qui est une actualisation et une amélioration de l'approche utilisée pour la base de données mondiale Geopolis (Moriconi-Ebrard, 1994), elle-même inspirée de celle de l'INSEE en France pour la définition des unités urbaines.

\section{Les agglomérations morphologiques de la base de données Brasipolis en 2010}

12 La base de données Geopolis définit comme urbaines toutes les agglomérations de plus de 10000 habitants, quelle que soit la définition officielle utilisée dans les pays. L'espace est considéré comme aggloméré s'il présente un ensemble continu de constructions, la continuité étant définie par une distance maximum de 200 mètres entre les constructions. Seule la continuité du tissu urbain importe et fait l'unité de l'agglomération, sans se soucier des limites administratives municipales. Ainsi, au Brésil, l'agglomération peut englober plusieurs chefs-lieux de municipios et de distritos, cidades et vilas. De plus, les cours d'eau traversés par un pont sont inclus dans ce calcul, ce qui, au Brésil, permet notamment de rattacher Niteroi à Rio de Janeiro de part et d'autre de l'entrée de la baie de Guanabara.

D'un côté, chaque agglomération morphologique a été vectorisée sous forme d'un polygone, en respectant ces règles et sur la base des images satellites de 2010. D'un autre côté, l'affectation des chiffres de population pour les agglomérations a été réalisée par superposition avec la cartographie des 316574 secteurs censitaires pour lesquels l'IBGE a publié les chiffres de population au recensement de 2010 
(illustration 2). Au final, seules les agglomérations de plus de 10000 habitants ont été retenues comme urbaines.

En 2010, Brasipolis identifie ainsi 1817 agglomérations urbaines et évalue le taux d'urbanisation à $78,3 \%$ contre $8,4 \%$ d'après les chiffres officiels. Cette différence n'est cependant qu'une moyenne agrégée au niveau du pays. Localement, des écarts encore plus importants avec les statistiques officielles sont relevés, conduisant à réévaluer tantôt à la hausse, tantôt à la baisse, certaines agglomérations comme dans les cas respectifs de São Luis ou de Esmeraldas cités précédemment. Cela conduit à réévaluer un grand nombre d'indicateurs relatifs à la primatie ou au positionnement réel dans la hiérarchie urbaine (illustration 3).

L'application de cette définition plus universelle de l'urbain permet de sortir de l'univers brésilien et de ses contraintes politiques pour appréhender un phénomène mondial. Elle permet également d'opérer des mesures justes et précises de surface, de densité, de distances, de forme etc. Cependant, seule l'extension des agglomérations était disponible pour la présente étude, la numérisation des extensions morphologiques des périodes antérieures étant en cours.

Illustration 2 - La méthodologie Brasipolis : l'exemple de l'agglomération urbaine de Joinville

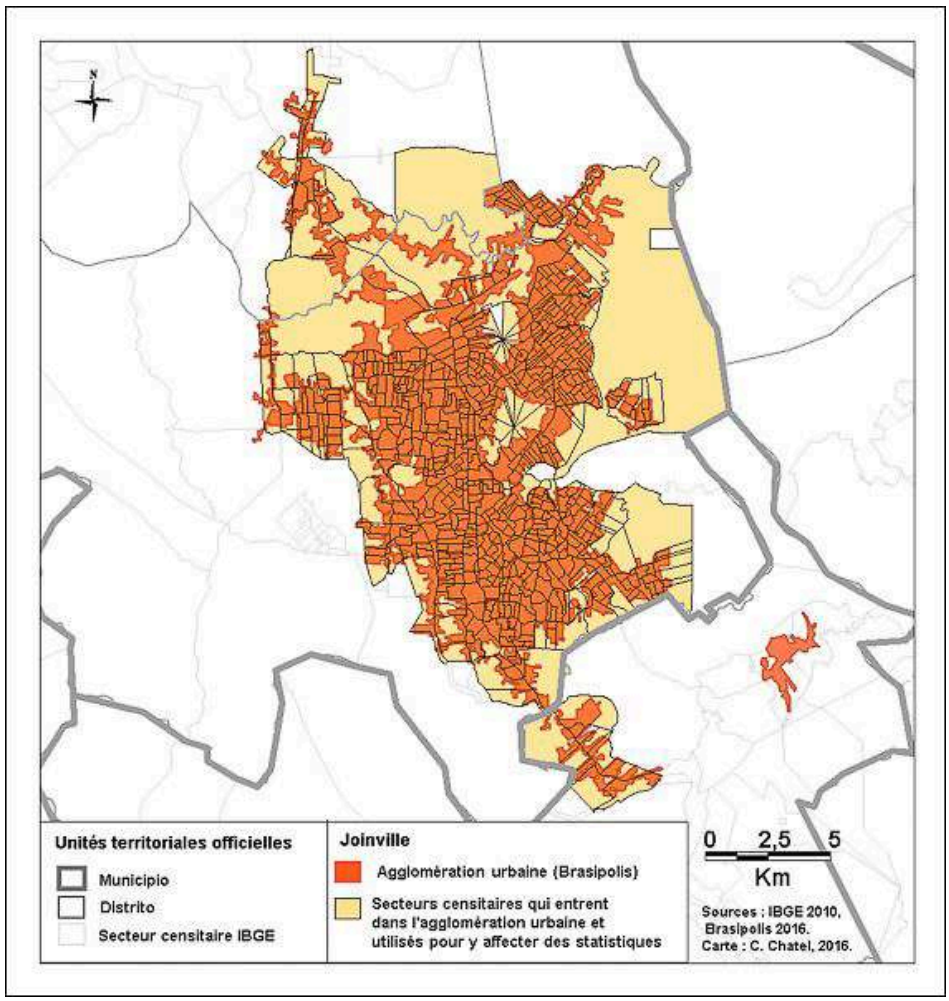


Illustration 3 - Les 40 agglomérations urbaines (Brasipolis) les plus peuplées du Brésil en 2010

\begin{tabular}{|c|c|c|c|c|c|c|c|c|c|}
\hline Rang & Ageglomération & Etat & $\begin{array}{l}\text { Population } \\
\text { 2mo }\end{array}$ & $\begin{array}{l}\text { Superficie } \\
\left(\ln ^{2}\right)\end{array}$ & Rang & Agejomenération & Etat & $\begin{array}{l}\text { Population } \\
\text { 2010 }\end{array}$ & $\begin{array}{l}\text { Superficie } \\
\left(\mathrm{Ln}^{2}\right)\end{array}$ \\
\hline 1 & São Paulo & SP & 18676973 & 2048 & 21 & São José dos Campos & SP & 885719 & 214 \\
\hline 2 & Rio de Janeiro & RJ & 11155037 & 1442 & 22 & Cuiabá & MT & 786190 & 173 \\
\hline 3 & Belo Horizonte & MG & 3924725 & 585 & 23 & Campo Grande & MS & 775859 & 190 \\
\hline 4 & Porto Alegre & RS & 3404316 & 682 & 24 & Aracaju & SE & 749225 & 94 \\
\hline 5 & Recife & $\mathrm{PE}$ & 3332083 & 417 & 25 & Sorocaba & SP & 714240 & 151 \\
\hline 6 & Fortaleza & CE & 3139367 & 402 & 26 & Florianópolis & SC & 707743 & 193 \\
\hline 7 & Salvador & $B A$ & 2987553 & 293 & $\boldsymbol{2 7}$ & Ribeirāo Preto & SP & 592070 & 139 \\
\hline 8 & Curitiba & $P R$ & 2567633 & 516 & 28 & Uberlândia & MG & 5863398 & 133 \\
\hline 9 & Campinas & SP & 2489620 & 786 & 29 & Londrina & PR & 578001 & 120 \\
\hline 10 & Brasilia & DF & 2478212 & 509 & 30 & Feira de Santana & BA & 532758 & 121 \\
\hline 11 & Belém & PA & 1982040 & 227 & 31 & Muiz de fora & MG & 506841 & 84 \\
\hline 12 & Manaus & AM & 1792881 & 203 & 32 & Koimille & sc & 488361 & 139 \\
\hline 13 & Goiânia & GO & 1755345 & 403 & $\mathbf{3 3}$ & Blumenau & SC. & 475166 & 251 \\
\hline 14 & Santos & SP & 1433004 & 243 & 34 & Maringá & PR & 4623886 & 114 \\
\hline 15 & São Luís & MA & 1292140 & 214 & 35 & Volta Redonda & RJ & 442145 & 69 \\
\hline 16 & Vitória & ES & 1084670 & 149 & 36 & Caxias do Sul & RS & 441753 & so \\
\hline 17 & Maceió & AL & 1001626 & 116 & $\mathbf{3 7}$ & Itajá & SC. & 401578 & 94 \\
\hline 18 & João Pessoa & PB & 981068 & 175 & $\mathbf{3 8}$ & Sāo losé do Rio Preto & SP & 387055 & 101 \\
\hline 19 & Teresina & $\mathrm{PI} / \mathrm{MA}$ & 905053 & 137 & 39 & Americana & SP & 385553 & 79 \\
\hline 20 & Natal & RN & 892547 & 143 & 40 & Campina Grande & PB & 374509 & 72 \\
\hline
\end{tabular}

\begin{tabular}{|l|l|l|l|l|l|}
\hline $\begin{array}{l}\text { Classe (1). } \\
\text { Métropole } \\
\text { internationale 1 }\end{array}$ & $\begin{array}{l}\text { Classe (5). } \\
\text { Satellites de la } \\
\text { métropole 1 }\end{array}$ & $\begin{array}{l}\text { Classe (2). } \\
\text { Métropole } \\
\text { internationale 2 }\end{array}$ & $\begin{array}{l}\text { Classe (3). } \\
\text { Métropole nationale }\end{array}$ & $\begin{array}{l}\text { Classe (4). } \\
\text { Métropole d'Etat }\end{array}$ & $\begin{array}{l}\text { Classes (6) à (11). } \\
\text { Autres villes }\end{array}$ \\
\hline \hline
\end{tabular}

Agglomérations urbaines selon la classification en entités urbaines définie pour cet article (paragraphe 3)

Source : Brasipolis, C. Chatel, 2016

Illustration 4 - Carte de situation des capitales des unités fédérales et des agglomérations urbaines (Brasipolis) les plus peuplées du Brésil en 2010

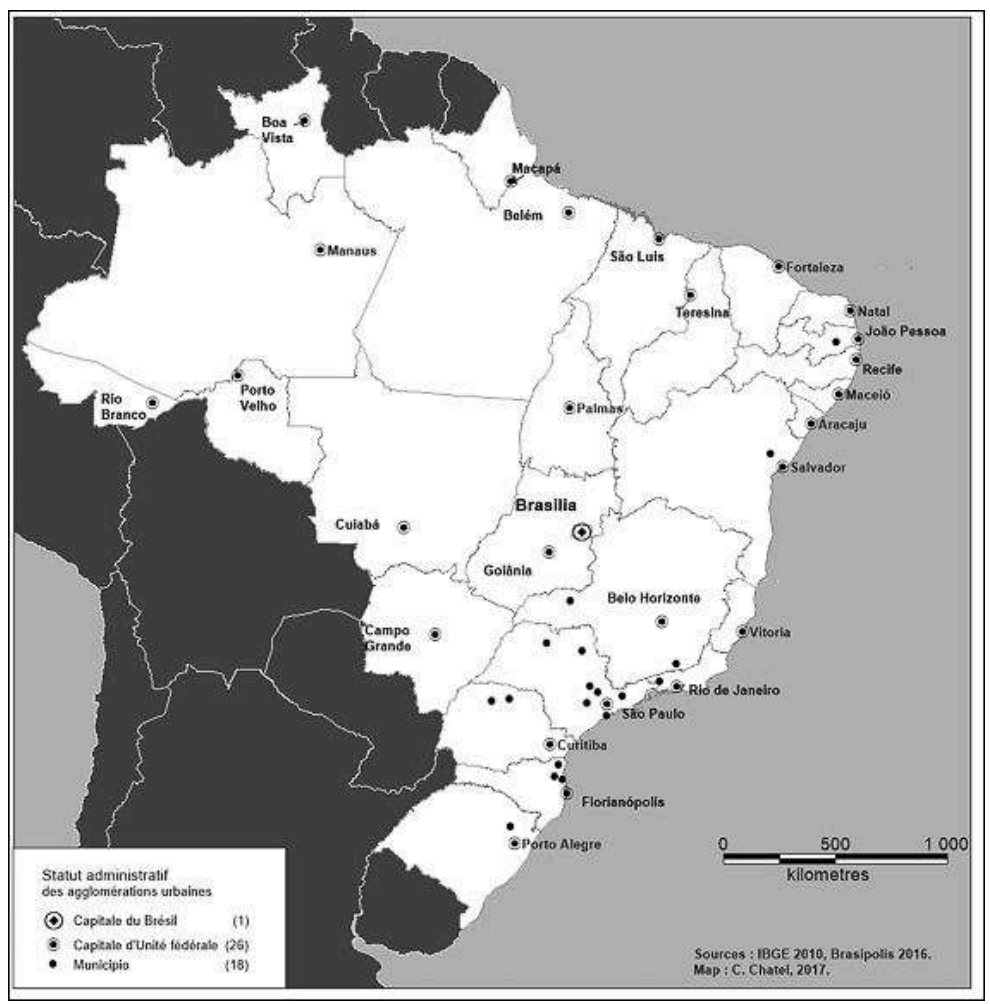




\section{Définitions et méthodologie : l'espace métropolitain et urbain}

\section{Définition de la population métropolitaine}

(bréliennes parce quelle se fonde dabord sur la spatialité de lagglomeration urbaine, et seulement dans un second temps sur les fonctions. Au contraire, la classification brésilienne REGIC (Regiões de Influência das Cidades) par exemple propose une hiérarchie fonctionnelle des centres basée sur leurs relations aux autres centres et aux territoires.

19 Le concept apparait pour la première fois dans le monde anglophone entre 1930 et 1940 lorsque l'on constate que certaines agglomérations ne se contentent pas de s'étaler, mais que populations et activités se reportent de plus en plus loin à la périphérie, dans des villes et villages sans continuité morphologique avec l'agglomération. La popularité de ce concept s'accroît après son utilisation par le Bureau of Census états-unien à l'occasion du recensement de 1950 (Klove, 1952). Ne pouvant plus s'appuyer sur la continuité du bâti, la définition vise à déterminer les limites d'une aire d'influence encore plus vaste que l'agglomération, à partir de l'intensité des flux polarisés, notamment les navettes domicile-travail. L'aire métropolitaine est ainsi définie comme un système régional de flux polarisés (Moriconi-Ebrard, 2000, p. 1). C'est sur cette base quelque peu floue que sont délimitées les megacities de l'ONU (cf. note 1, supra), qui se garde bien d'avancer des critères chiffrés.

Certains pays appliquent de véritables critères statistiques, tels les États-Unis (standard metropolitan areas -SMA). En France, cette notion renvoie au zonage statistique en aires urbaines créé en 1999, le mot métropolitain étant depuis 1966 réservé officiellement aux capitales des régions désignées comme métropoles d'équilibre. L'extension d'une aire urbaine française est définie par un ensemble de critères qui permettent d'ajouter à une agglomération - appelée unité urbaine par l'INSEE - qui offre un nombre minimum d'emplois - appelée alors pôle urbain -, les communes alentour dont au minimum $40 \%$ des actifs travaillent dans le pôle ou dans les communes attirées par celui-ci ${ }^{4}$.

21 La notion d'aire métropolitaine associe également une idée de dimensions supérieures à celles de l'agglomération urbaine. Ainsi, aux États-Unis l'agglomération urbaine (urban cluster) est définie jusqu'au seuil minimum de 2500 habitants, tandis que les aires métropolitaines doivent en compter au moins 50000 (SMA). La plupart des pays réservent cette notion à des masses démographiques supérieures à 1 million d'habitants (Corée du Sud, Afrique du Sud, Mexique etc.), voire tout simplement à la seule capitale nationale - la métropole nationale s'opposant à la province : c'est le cas de l'Argentine, de Taiwan, de la Thaïlande, des Philippines, ou de la France d'avant 1966.

EchoGéo, 41 | 2017 
$22 \mathrm{Au}$ Brésil, la notion correspondante est appelée région métropolitaine (região metropolitana). Jusqu'à l'approbation de la Constitution brésilienne de 1988, la définition des régions métropolitaines relevait de la compétence du Congrès National. Elle s'appuyait sur des études scientifiques, même si elle pouvait être influencée par des intérêts politiques. Depuis 1988, le paragraphe 3 de l'article 25 de la Constitution donne cette attribution aux assemblées législatives des États de la fédération. Dans la plupart des cas, les États décident les limites des régions métropolitaines sans appui technique (Fernandes, Araújo, 2013). Ainsi, cette définition n'est ni statistique, ni nationale. Par exemple, le Santa Catarina, qui est le seul État à ne pas présenter de primatie en faveur de la capitale, est paradoxalement le seul à être couvert à $100 \%$ par des aires métropolitaines.

23 La classification REGIC citée ci-avant, n'est qu'une étude des aires métropolitaines et urbaines parmi d'autres au Brésil. Depuis les années 1960, plusieurs études ont identifié des métropoles ou des territoires urbains autour d'agglomérations grandes ou moins grandes pour démontrer l'effet de polarisation exercé par les villes sur leurs périphéries. Ce sont d'un côté les régions d'influence des villes, Regiões de Influência das Cidades (REGIC), appelées ainsi depuis l'étude de 1978, mais appelées auparavant espaços homogênenos e espaços polarizados (1967, Espaces homogènes et espaces polarisés), microregiões homogêneas (1968, Micro-régions homogènes), regiões funcionais urbanas (1972, Régions urbaines fonctionnelles) (Ministerio..., 2008); et d'un autre côté, les Arranjos populacionais e concentrações urbanas do Brasil (Groupement de population et concentrations urbaines du Brésil), qui ont reçu d'autres noms dans les études précédentes: Áreas metropolitanas en 1969; aglomerações urbanas en 1975, Áreas de concentração de população en 2006 (Ministerio..., 2015).

On perd peu à peu de vue la caractéristique de concentration massive et de complexité fonctionnelle, ainsi que sociale et politique qui caractérise ces espaces, qui est au centre de notre question, tandis que ces définitions nous confinent dans une approche de la métropole relative au seul contexte brésilien.

Une seconde préoccupation très générale relative aux métropoles concerne l'étalement urbain, appelé au Brésil dispersão urbana. (Soja, 2006; Reis, 2007, Sposito, 2009). On remarque ici un renversement de paradigme. Autrefois, la littérature présentait la croissance urbaine comme le fruit de l'exode rural, donc de mouvements centripètes. Or, avec l'aire métropolitaine, la croissance est au contraire décrite comme le résultat d'un desserrement, donc de mouvements centrifuges. L'étalement est alimenté de l'intérieur, par dé-densification du noyau de l'agglomération.

Selon les diverses études citées ci-dessus, le Brésil compte incontestablement un grand nombre de métropoles de différents niveaux. Néanmoins, seulement deux d'entre elles se détachent des autres dans la hiérarchie nationale : São Paulo et Rio de Janeiro, qui sont les megacities de l'ONU. Plutôt que de choisir parmi la profusion de classifications proposées par l'IBGE, nous avons ici opté pour une méthode conditionnée par la possibilité de construire des séries diachroniques d'évolution démographique.

\section{Méthodologie employée : les entités urbaines définies à partir des agglomérations de Brasipolis}

27 La question initiale de la recherche n'étant pas de savoir si les aires urbaines et métropolitaines s'étalent, mais au contraire de vérifier si la population s'accumule 
aujourd'hui au détriment des autres villes, c'est en partant d'une extension fixe et actuelle des agglomérations urbaines que l'on peut répondre à cette question.

Les agglomérations morphologiques constituent la base de l'échantillon. Pour obtenir un pavage exhaustif du territoire, on a simplement étendu chaque agglomération identifiée en 2010 dans le cadre du programme Brasipolis, aux municipios entiers sur lesquels elles s'étendent. Ce sont donc, comme dans le cas des unités urbaines françaises de l'INSEE, des agglomérations ajustées sur les contours municipaux, sauf que les municipios brésiliens sont beaucoup plus vastes qu'une commune française. Les municipalités formant la même agglomération sont de ce fait considérées ensemble comme une seule et même entité. Une entité sera dite urbaine lorsqu'elle contient au moins une agglomération de plus de 10000 habitants.

On obtient 1734 entités urbaines, impliquant 1934 municipios. 61 entités s'étendent sur plus d'une municipalité, la grande majorité - 1673 - n'étant constituée que d'un seul municipio. Le complément de la population du Brésil, soit 3633 municipalités en 2010, est considéré comme non urbain dans cette étude, car aucune agglomération de 10000 habitants n'y a été identifiée.

Pour chaque entité urbaine, la population est restituée pour les recensements de 1900, 1920, 1940, 1950, 1960, 1970, 1980, 1991, 2000 et 2010, et estimée par l'IBGE en 2016.

\section{São Paulo et Rio de Janeiro}

31 Les agglomérations ne cessent de s'étendre et ce phénomène est particulièrement intense autour des megacities. Une mise à jour effectuée en 2016 montre que les agglomérations de Campinas, São José dos Campos et de Sorocaba, qui étaient encore distinctes en 2010 (Chatel et al., 2015a, 2015b), sont désormais réunies à l'agglomération de São Paulo en 2016 (illustration 4). En ajoutant l'agglomération de Santos, située sur le littoral à 700 mètres en contrebas de São Paulo, on distingue un ensemble qu'on appellera la région métropolitaine étendue de São Paulo (RMESP). Cette région ne correspond à aucune définition officielle, mais à l'exception de Santos, à un bloc aggloméré d'un seul tenant en 2016 ajusté sur 29 municipios entiers, dont le déploiement a rayonné à partir de la capitale de l'État le plus riche et le plus peuplé du Brésil. La densité de population, d'infrastructures et de richesse (Sposito, 2005) est exceptionnelle dans le continent et dans le monde. 
Illustration 5 - São Paulo : région métropolitaine étendue (2016), agglomérations ajustées sur les municipios et agglomérations (2010)

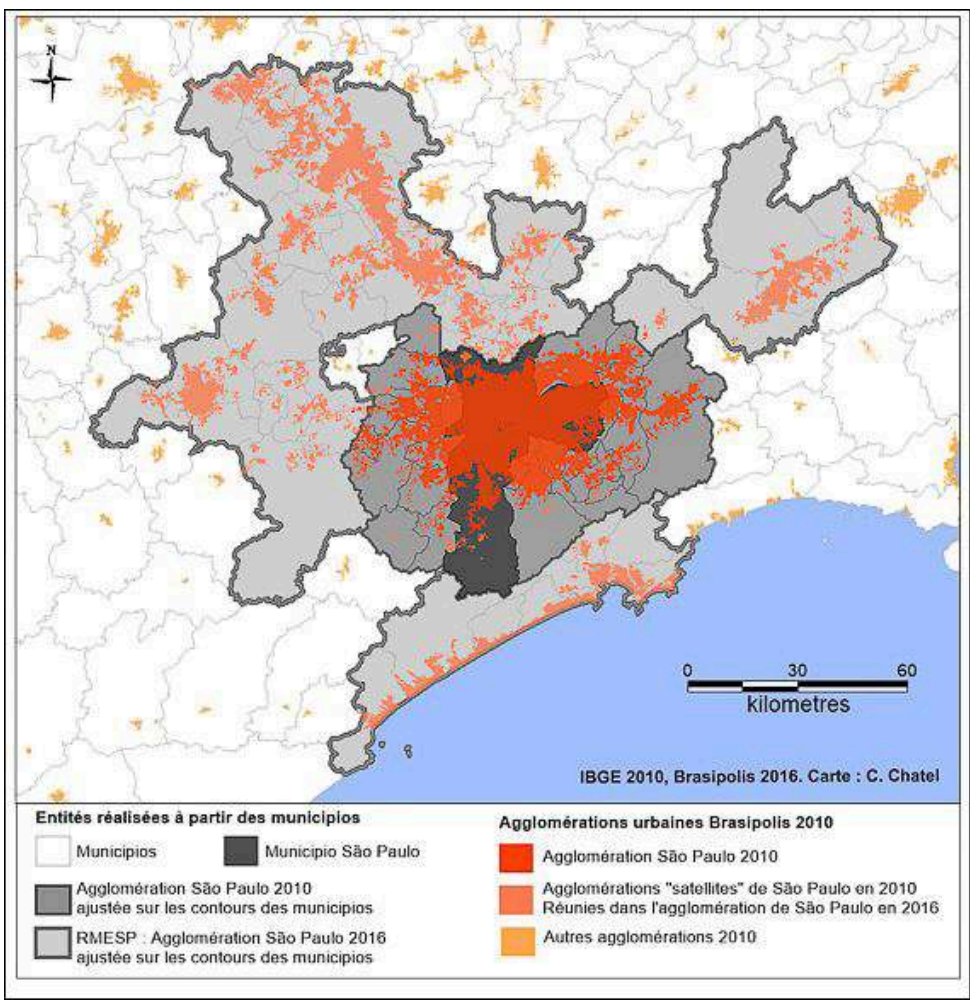

millions habitants vivent, travaillent et se déplacent quotidiennement dans cet espace de $17580 \mathrm{~km}^{2}$, soit une densité résidentielle de 1593 habitants au $\mathrm{km}^{2}$, digne des deltas du Nil et de l'Asie du Sud-Est.

Le caractère méta-urbain de ce type d'organisme spatial conduit à ajouter les millions de personnes qui transitent par les plaques tournantes nationales et internationales de ses trois aéroports et par ses immenses gares routières, ainsi que des millions de personnes de passage pour affaire ou en visite touristique. Ceci différencie radicalement la RMESP des plaines agricoles d'Asie du Sud-Est.

En Europe ou aux États-Unis, il n'est pas possible de combiner à la fois une densité aussi élevée et une telle masse de population. À l'échelle planétaire, seuls le Kanto (région de Tokyo) et deux régions de Chine (Moriconi-Ébrard et al., 2017), à la fois urbaines et industrielles, surpassent la RMESP en termes de concentration. On se trouve donc face à un objet géographique qui, sans être unique, peut être objectivement qualifié de très rare à l'échelle mondiale.

La région métropolitaine étendue de São Paulo a ainsi connu un accroissement démographique annuel de $0,82 \%$ entre 2010 et 2016, ce qui représente 230000 habitants supplémentaires chaque année : c'est presque l'équivalent de celui de l'agglomération entière de Brasília en 10 ans.

Avec 0,31 \% depuis 2010, le taux de croissance de l'agglomération de Rio de Janeiro, est encore plus faible. Comparée à São Paulo, elle a engendré des extensions spatiales bien plus modestes depuis le dernier recensement (illustration 5). L'ensemble ajusté sur les contours des municipios en 2016, la région métropolitaine étendue de Rio de Janeiro (RMERJ) rassemble néanmoins 13,1 millions d'habitants en 2016 sur $8816 \mathrm{~km}^{2}$, soit une 
densité de 1483 habitants au $\mathrm{km}^{2}$. Ces dimensions sont comparables à des métropoles comme Moscou, Istanbul ou Buenos Aires.

Illustration 6 - Rio de Janeiro : région métropolitaine étendue (2016), agglomérations ajustées sur les municipios et agglomérations (2010)

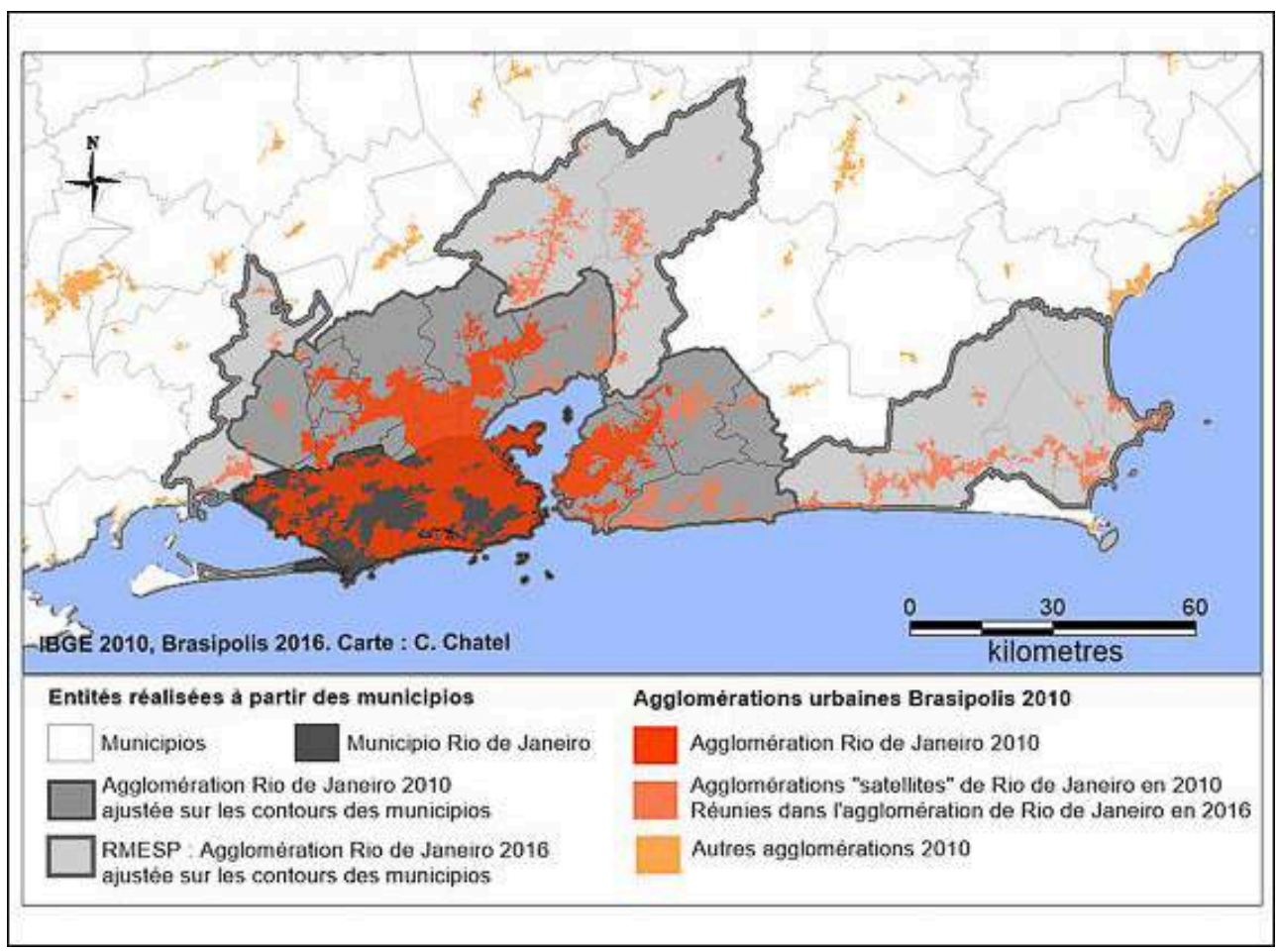

\section{Typologie des trajectoires et résultats}

\section{Une approche multicritère}

La classification des trajectoires de la croissance des 1734 entités urbaines, c'est-à-dire des agglomérations de Brasipolis ajustées sur les municipios entiers, fait apparaître plusieurs facteurs correspondant à trois axes, chacun étant considéré toutes conditions égales par ailleurs.

Le premier groupe procède d'une logique aspatiale, dérivant de la taille de l'agglomération urbaine en 2010. Le second est au contraire purement spatial car lié à la proximité d'une megacity : il est en fait constitué des agglomérations de la périphérie de São Paulo qui se sont agglomérées à cette dernière entre 2010 et 2016. Le troisième groupe fait émerger un facteur qualitatif. Il procède d'une logique politique au plan fédéral propre à l'organisation du Brésil : le fait d'être capitale d'État ou non.

On calcule ensuite l'évolution de la population des agglomérations urbaines entre 1900 et 2016 en se basant sur les populations ajustées sur les municipios entiers. On peut distribuer les résultats en 11 classes, auxquelles s'ajoute la classe des municipalités dites non urbaines selon notre approche.

- Classes (1) et (2). Les megacities de São Paulo et Rio de Janeiro affichent chacune une trajectoire de croissance très différente. 
- Classe (3). 7 capitales d'État dont les agglomérations atteignent entre 2,5 et 4 millions d'habitants en 2016. Elles sont à raison considérées comme de grandes métropoles du Brésil, mais de stature nationale ou macro-régionale, et non pas internationale. En référence à une interprétation classique des distributions parétiennes (illustration 6), le fait que ce groupe soit très peu hiérarchisé indique que, derrière São Paulo et Rio de Janeiro, ces agglomérations entretiennent entre elles un rapport de concurrence plutôt que hiérarchique à l'échelle nationale. Peu d'auteurs sont d'ailleurs d'accord pour désigner quelle est la troisième ville du pays. En termes de municipalités, il s'agit de Salvador ; d'agglomérations, de Belo Horizonte ; de fonctions politiques, de Brasília. Mais, en fonction d'autres critères, tels que l'IDH, la richesse ou le rayonnement politique et intellectuel, on peut aussi citer Recife, Fortaleza, Porto Alegre, voire Curitiba. De plus ce classement interne varie au fil des années, tandis que le rang des deux megacities n'est jamais remis en question.

- Classe (4). Cette classe politico-administrative est formée de 18 capitales d'État centres d'agglomérations de moins de 2 millions d'habitants en 2010. Les moins peuplées sont les plus récentes. La plus petite, Palmas, capitale de l'État du Tocantins, compte 217000 habitants en 2010, mais déjà plus de 320000 en 2016.

- Classe (5). Les satellites de São Paulo, avec pas moins de 4 agglomérations de 700000 à 2,5 millions d'habitants, ainsi que 8 autres de 100000 à 400000 habitants présentent une trajectoire typique des périphéries d'une aire métropolitaine.

- Les classes (6) à (11) sont les entités urbaines qui n'entrent pas dans les classes précédentes, et qui se déclinent simplement en continu par tranches de taille, entre 10000 et 587000 habitants.

- La classe (12) correspond aux municipalités considérées comme non urbaines.

Illustration 7 - Distribution rang-taille des agglomérations urbaines du Brésil

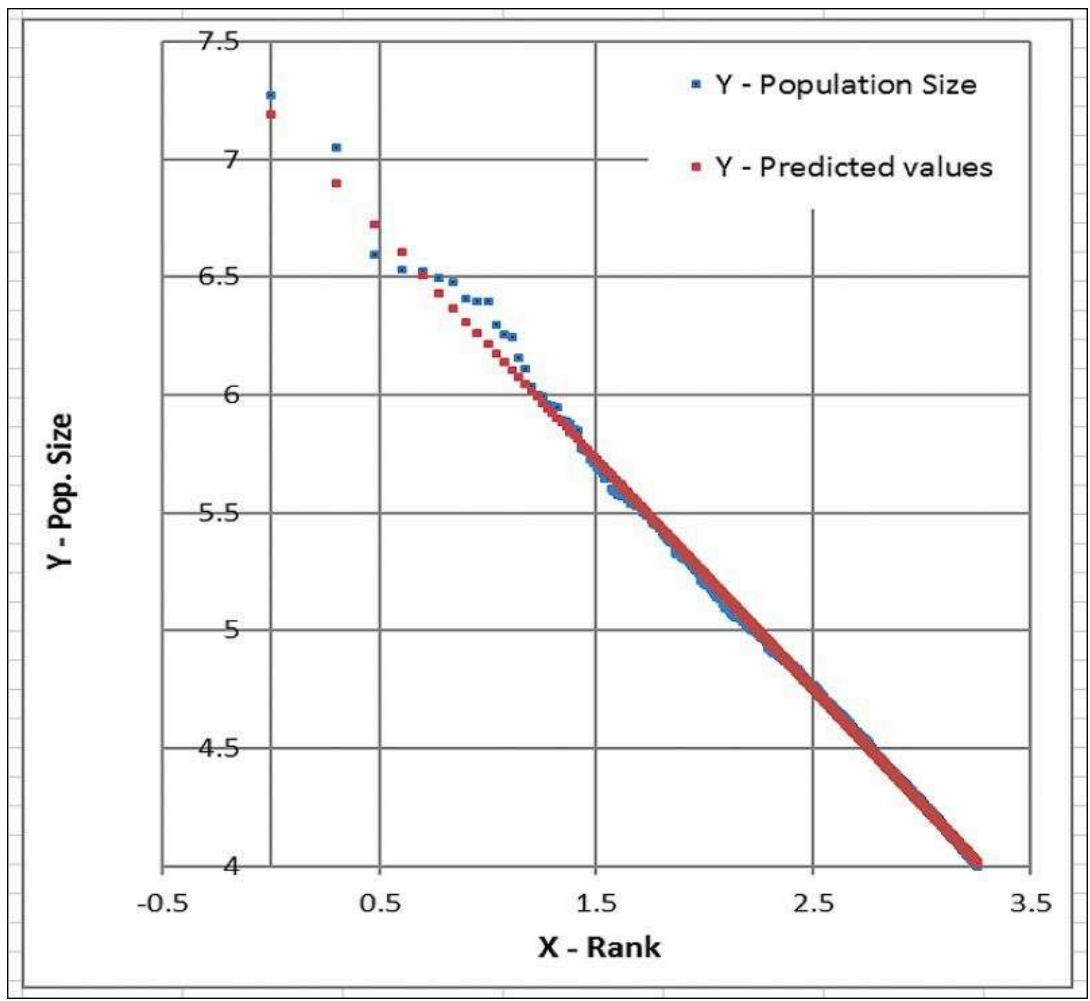




\section{Remarques générales}

Pour interpréter ces résultats (illustration 7) deux observations préliminaires s'imposent.

- un phénomène bien connu et que nous avons déjà cité est à garder en mémoire: le ralentissement de la croissance démographique du Brésil, qui a chuté des deux tiers depuis les années 1950.

- la convergence des taux de variation entre les différentes classes est remarquable. À partir des années 1980, l'écart-type entre les classes chute, non seulement en valeur absolue, mais surtout en proportion de la moyenne dont il ne représente plus que $30 \%$, alors que la croissance du Brésil a elle-même chuté des deux tiers. Cette convergence concerne de plus toutes les classes: les extrêmes le sont de moins en moins. Ce phénomène décrit un aplanissement spectaculaire des disparités liées à la taille des agglomérations, laquelle semble de ce fait de moins en moins déterminante face au développement.

41 Cette convergence se traduit sur les cartes par une diffusion de plus en plus large des municipios dont la croissance est supérieure à la moyenne nationale, qu'ils soient urbains ou non (illustration 8).

Tableau 8- Taux de variation annuel moyen de la population suivant 12 classes d'entités

\begin{tabular}{|c|c|c|c|c|c|c|c|c|c|c|}
\hline Entités urbaines et rurales par classes & \begin{tabular}{|l}
$1900 \AA$ \\
1920 \\
\end{tabular} & \begin{tabular}{|l}
$920 \mathrm{a}$ \\
1940 \\
\end{tabular} & \begin{tabular}{|l}
$1940 \mathrm{a}$ \\
1950 \\
\end{tabular} & $\begin{array}{l}1950 \text { aे } \\
1960 \\
\end{array}$ & \begin{tabular}{|l}
$1960 \mathrm{a}$ \\
1970 \\
\end{tabular} & \begin{tabular}{|l}
970 à \\
1980 \\
\end{tabular} & \begin{tabular}{|l}
$1980 \mathrm{a}$ \\
1990 \\
\end{tabular} & $\begin{array}{l}1990 \mathrm{~d} \\
2000\end{array}$ & \begin{tabular}{|l|l|}
$2000 \mathrm{i}$ \\
2010
\end{tabular} & \begin{tabular}{|l|l|l}
$2010 \mathrm{a}$ \\
2016 \\
\end{tabular} \\
\hline 1-Sao Paulo & 4,51 & 4,93 & 5,52 & 6,14 & 5,44 & 4,46 & 2 & 1,58 & 0,94 & 1,08 \\
\hline 2- Rio de Janeiro & 2,22 & 3,27 & 3,59 & 4,38 & 3,52 & 2,42 & 1,11 & 1,17 & 0,83 & 0,44 \\
\hline 3- Capitales de plus de 2,5 millions dhabitants & 4,63 & 3,84 & 3,72 & 5,73 & 5,1 & 4,52 & 2,93 & 2,13 & 1,23 & 1,21 \\
\hline 4- Capitales de moins de 2 millions d'habitants & 3,78 & 2,49 & 2,38 & 4,46 & 5,28 & 5,43 & 4,53 & 2,9 & 1,96 & 1,54 \\
\hline 5- Satellites São Paulo & 2,9 & 2,65 & 2,05 & 4,19 & 4,48 & 5,06 & 3,32 & 2,27 & 1,47 & 1,39 \\
\hline 6. de 400000 à 587000 habitants & 2,48 & 1,45 & 2,43 & 5,45 & 3,54 & 4,11 & 3,2 & 2,18 & 1,69 & 1,46 \\
\hline 7 - de 200000 a 399999 habitants & 3,78 & 1,87 & 1,63 & 3,27 & 2,76 & 3,78 & 2,46 & 1,85 & 1,26 & 1,12 \\
\hline 8. de 100000 \& 199999 habitants & 3,55 & 1,19 & 2,21 & 1,97 & 1,46 & 2,93 & 2,46 & 1,73 & 1,62 & 1,37 \\
\hline 9- de 50000 a 99999 habitants & 3,47 & 1,33 & 1,4 & 2,23 & 1,36 & 2,58 & 2,43 & 1,42 & 1,42 & 1,28 \\
\hline 10 - de 20000 a 49999 habitants & 3,44 & 1,11 & 1,62 & 0,99 & 0,89 & 1,75 & 1,66 & 0,76 & 1,16 & 1,09 \\
\hline 11 - de 10000 a 19999 habitants & 3,89 & 1,73 & 1,81 & 2,05 & 0,7 & 1,06 & 1,17 & 0,71 & 1,01 & 1,03 \\
\hline 12 - Entités non urbaines (moins de 10000 habitants) & 6,6 & 0,36 & 2,87 & 4,54 & 4,05 & 0,25 & 1,25 & 1,84 & 0,6 & 0,55 \\
\hline Brésil entier & 3,49 & 1,5 & 2,31 & 3,15 & 2,82 & 2,48 & 2,12 & 1,63 & 1,17 & $J, H$ \\
\hline Ecarn-type des 12 classes & 1.14 & 1,31 & 1,18 & 1,66 & 1,75 & 1,63 & 1,02 & 0,64 & 0,39 & 0,34 \\
\hline Ecarn-type exprimé en \% de la moyenne & $33 \%$ & $87 \%$ & $51 \%$ & $53 \%$ & $62 \%$ & $66 \%$ & $48 \%$ & $39 \%$ & $33 \%$ & $30 \%$ \\
\hline
\end{tabular}

\begin{tabular}{|c|c|c|c|c|c|c|}
\hline \multicolumn{3}{|c|}{ croissance inférieure à celle du Brésil $\longleftarrow$} & \multicolumn{4}{|c|}{$\longrightarrow$ croissance supérieure à celle du Brésil } \\
\hline-2.00 & -1.00 & 0.00 & 0.00 & 1.00 & 2.00 & 3.00 \\
\hline
\end{tabular}

Légende 
Illustration 9 - Taux de variation annuel moyen par municipios comparé au taux de variation annuel moyen au Brésil (1900-2016)

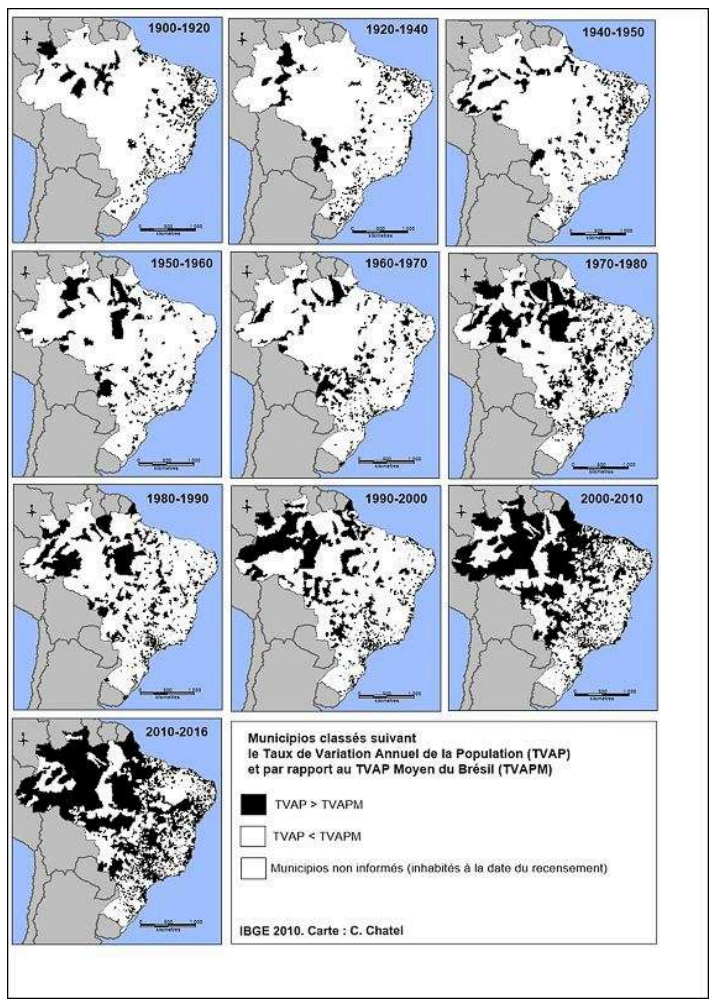

\section{Caractéristique des trajectoires}

\section{La baisse de la croissance des megacities}

L'agglomération actuelle de São Paulo a été une aire extrêmement dynamique jusqu'en 1980 , atteignant $+6,14 \%$ par an durant la décennie 1950-1960, ce qui la situait à l'époque parmi les records mondiaux. Il est donc curieux de noter que la croissance des megacities n'est perçue comme prédatrice... qu'au moment où leur croissance s'effondre.

Les années 1980-1990 se caractérisent par une chute brutale du rythme de la croissance des deux grandes métropoles internationales. En 2016, la croissance de la population de Rio de Janeiro, qui a perdu ses fonctions de capitale nationale au profit de Brasilia en 1960, a été divisée par 10 depuis les années 1950. L'agglomération de São Paulo suit le même mouvement, avec 10 à 20 ans de décalage: sa croissance est inférieure à la moyenne nationale depuis près de 40 ans, laquelle a elle-même chuté de moitié. Le développement des immenses agglomérations satellites de la périphérie de São Paulo (classe 5), aujourd'hui intégrées dans la nouvelle agglomération, ne parvient même pas à compenser le déclin relatif de la RMESP dans son ensemble (classes 1+5). Suivant un hypothétique scénario de prédation du territoire, c'est dans la classe (5), les satellites de São Paulo, que l'on devrait observer les records de croissance au Brésil. Or, dès les années 1980 on reste en dessous de la croissance des capitales d'État de taille moyenne (de moins de 2 millions d'habitants, classe 4). De plus cette croissance se tasse lentement au fil des décennies. 


\section{Le triomphe des capitales d'État de taille modeste} d'État de taille moyenne (classe 4). Dès les années 1960, elles flirtent avec le record de l'époque, la métropole pauliste. Leur croissance est restée supérieure à toutes les autres catégories jusqu'à nos jours. Il est remarquable qu'elle soit constamment supérieure :

- à la croissance des satellites de São Paulo, à l'exception des années 1920-1930, qui profitent pourtant directement du desserrement de la megacity toute proche ;

- à la croissance des grandes capitales d'État (classe 3, dotées d'une agglomération de 2,5 à 4 millions d'habitants) et ce, malgré l'introduction dans cet échantillon d'un élément particulièrement vigoureux: l'agglomération de Brasília, partie de rien en 1959, et qui atteint déjà 3 millions d'habitants en 2016.

Si les agglomérations de la classe 3 sont héritées de l'«archipel des métropoles » de la période coloniale (Théry, 1985), ce n'est pas le cas de celles de la classe 4, qui sont en majorités des créations plus récentes de l'intérieur du Brésil. Une nouvelle fois, on note une forte convergence, qui se traduit sur la carte nationale par une forte primatie des réseaux urbains des États de la fédération (carte 5).

Illustration 10 - Primatie et macrocéphalie par État au Brésil en 2010

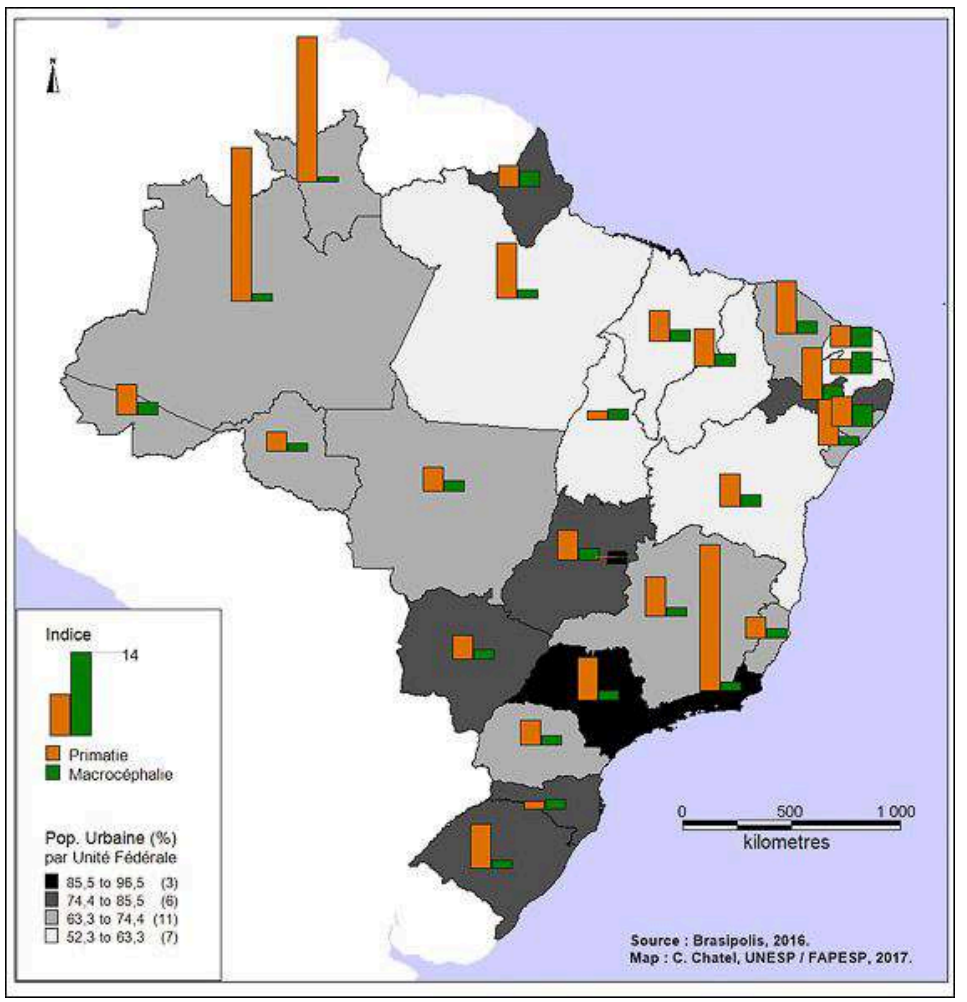

\section{Un attracteur lié à la taille de l'agglomération?}

Depuis 1980, la croissance de cette classe 3 est inférieure à celle des agglomérations de 400000 à 587000 habitants sans fonction politique de capitale d'État (classe 6). La tendance à la concentration de la population dans les megacities voire dans les grandes métropoles nationales brésiliennes, n'est plus d'actualité du point de vue relatif des taux de croissance relevés. 
47 Ce phénomène apparaît également dans les classes suivantes (de 7 à 11) : les agglomérations de 10000 à 400000 habitants, forment un bataillon de 1682 agglomérations qui évolue presque constamment en dessous de la moyenne nationale. Or la situation se retourne, encore en 1970, en faveur des plus de 50000 habitants (classes 7, 8, 9).

48 Il semble exister un attracteur lié à la taille des agglomérations L'optimum de taille se trouve plutôt dans les agglomérations secondaires comptant entre 50000 et 2 millions d'habitants en 2010, avec un pic entre 400000 et 600000 habitants, incluant les capitales d'État de cette taille.

\section{Deux conclusions}

Aux antipodes des intuitions véhiculées par une pratique du terrain, la croissance des grandes métropoles est relativement en perte de vitesse, depuis déjà un demi-siècle. S'il existe un attracteur lié à la taille des agglomérations brésiliennes, il se situe actuellement plutôt autour de 400 à 600000 habitants, masse démographique qui n'a en soi encore rien de préoccupant. En effet, selon la taille des villes, la dynamique démographique a des conséquences très différentes en termes d'aménagement, de gestion des ressources, de gouvernance, de quotidien pour les habitants. Même avec des variations de population inférieure à la moyenne nationale, les métropoles conserveront pour longtemps ce «coup d'avance" par rapport aux autres villes. Néanmoins, la dynamique des capitales d'État, notamment de l'intérieur du Brésil, et des villes moyennes en général, introduit depuis quelques décennies un rééquilibrage progressif de la distribution de la population dans ce vaste pays.

50 Les trajectoires que nous avons décrites attirent aussi l'attention sur le rôle du politique de deux façons. Tout d'abord, sur le statut de capitale d'État, qui rend n'importe quelle ville attractive. Ensuite sur les inflexions des courbes qui montrent la coïncidence entre les périodes de changement de régime de la croissance et les changements de régimes politiques. Le succès des capitales d'État est clairement lié au passage à la dictature militaire (1964-1985). Partant, on ne peut guère parler de décentralisation, mais plutôt de caporalisation du système urbain, analogue au phénomène décrit à propos de la Russie soviétique (Moriconi-Ebrard, 2004).

51 Le fait que ce phénomène se poursuive avec le retour à la démocratie n'a rien d'étonnant. En fait, il n'a aucune raison de changer car les équipements, les circuits d'échange et de décision, les structures administratives lourdes, les acteurs de l'économie ont lancé un processus dont l'inertie spatiale dépasse la labilité des gouvernements. En clair, le retour à la démocratie n'apparaît pas comme une révolution, mais comme une réappropriation des structures de la gouvernance et des outils de l'aménagement.

52 La seconde conclusion tente de répondre, quant à elle, à une inquiétude sous-jacente et largement partagée hors des frontières du Brésil, comme en témoignent les Conférences UN Habitat (Vancouver, 1976 ; Istanbul, 1996 ; Quito, 2016) : la croissance des grandes métropoles semble ne devoir rencontrer aucune limite (Moriconi-Ebrard et al., 2017). Cette question doit se poser dans un registre non plus relatif à la mécanique des lois parétiennes qui régulent la dynamique d'un système urbain, mais à l'échelle humaine. Ici, la réponse est également claire : la population de la région métropolitaine 
étendue de São Paulo (RMESP) telle que nous l'avons identifiée croît de 0,82 \% par an. Cela représente 13 personnes supplémentaires par $\mathrm{km}^{2}$ dans un espace qui en compte déjà 1593 en moyenne. 13 personnes réelles et visibles, et non pas une catégorie abstraite des statistiques. 13 personnes, surtout, avec leurs activités, leur mobilité quotidienne, les services et les équipements qu'elles utilisent, ainsi que les biens qu'elles possèdent en quantité toujours plus grande, puisque le niveau de vie moyen individuel et la production n'ont cessé d'augmenter (Sposito, 2016).

Ces deux réponses ont au moins le mérite de réveiller un intérêt d'ordre herméneutique : elles ne s'opposent pas entre elles mais doivent se poser côte à côte.

\section{BIBLIOGRAPHY}

Bruegmann R, 2005. Sprawl : A compact History. Chicago, University of Chicago Press, 309 p.

Chatel C., Sposito M. E. B, 2015a. Forma e expansão urbana no Brasil: fatos e hipóteses. Primeiros resultados do banco de dados BRASIpolis. Cidades, vol. 12, n 21, p. 108-153.

Chatel C., Sposito M. E. B, 2015b. Forme et étalement urbain au Brésil : faits et hypothèses. Premiers enseignements de la base de données BRASIpolis. Cidades, vol. 12, n 21, p. 153-197.

Fernandes A.S.A, Araújo S.M.V, 2015. A criação de municípios e a formalização de regiões metropolitanas: os desafios da coordenação federativa. Urbe. Revista Brasileira de Gestão Urbana (Brazilian Journal of Urban Management), 7(3), p. 295-309.

Klove R., 1952. The Definition of Standard Metropolitan Areas. Economic Geography, 28(2), p. 95-104.

Ministério do Planejamento, Orçamento e Gestão, IBGE, Diretoria de Geociências, Coordenação de Geografia, 2008. Regiões de Influência das Cidades 2007, Rio de Janeiro, IBGE, 201 p.

Ministério do Planejamento, Orçamento e Gestão, IBGE, Diretoria de Geociências, Coordenação de Geografia, 2015. Arranjos Populacionais e Concentrações Urbanas do Brasil, Rio de Janeiro, IBGE, 167 p.

Moriconi-Ebrard F., 1994. Géopolis : pour comparer les villes du monde. Paris, Ed. Economica, Anthropos, Coll.Villes.

Moriconi-Ebrard F., 2000. De Babylone à Tokyo, les grandes agglomérations du Monde. Paris, Ophrys, Coll. GéOphrys, 344 p.

Moriconi-Ebrard F., 2004. La "caporalisation" des réseaux urbains dans les pays de l'Est. Regard sur l'Est, Dossier n 36 « Capitales de l'Est, sous le feu des lumières !».

Moriconi-Ebrard F., Perez J., 2017. Shanghai et Guangzhou sont les deux agglomérations urbaines les plus peuplées du monde. Confins, 30/ 2017.

Reis F. N. G., Tanaka M.S., 2007. Brasil: estudos sobre dispersão urbana. São Paulo, Via das Artes/ FAUUSP.

Soja E. W., 2006. Algunas consideraciones sobre el concepto de ciudades-región globales. Cadernos IPPUR, Rio de Janeiro, v. 20, n. 2, p. 9 - 44. 
Sposito M. E. B., 2005. O chão em pedaços: urbanização, cidades e economia no Estado de São Paulo. Presidente Prudente: Faculdade de Ciências e Tecnologia/UNESP, tese de livre docência.

Sposito M. E. B., 2009. Urbanização difusa e cidades dispersas: perspectivas espaço-temporais contemporâneas. In Reis F. N. G., Sobre dispersão urbana, São Paulo, Via das Artes, p. 35-54.

Sposito M. E. B., 2016. Reestruturação urbana e transformações nas cidades médias: consumo e cotidiano. In III Simpósio Internacional sobre Metropolização do Espaço, Gestão Territorial e Relações Urbano-Rurais, Rio de Janeiro: Pontifícia Universidade Católica do Rio de Janeiro, v. 1. p. 1-4

Théry H., 1985. Le Brésil, Collection U, Armand Colin, Paris, 231 p.

\section{NOTES}

1. Au sens de l'ONU : agglomérations de plus de 10 millions d'habitants.

2. Ce rythme d'accroissement est à peu près celui de la population globale actuelle du Brésil.

3. Institut Brésilien de Géographie et de Statistiques.

4. Voir la définition de l'aire urbaine de l'INSEE. https://www.insee.fr/fr/metadonnees/ definition/c2070 .

\section{ABSTRACTS}

Huge demographic and land tenure pressures Brazil's two metropolis: São Paulo and Rio de Janeiro. However, the growth of these two metropolis has been steadily declining for half a century. It is now much lower than national growth. The methodology used consists in reconstructing the entire urban hierarchy of the country, starting from a strictly morphological definition of the urban agglomerations. This approach allows to verify that there is indeed an "attractor" of demographic size which is currently around 500000 inhabitants. It also highlights the role of territorial administrative and political functions, notably when the cities are a state capital.

Une pression démographique et foncière énorme pèse sur les deux métropoles du Brésil : São Paulo et Rio de Janeiro. Or, le rythme de la croissance de ces deux métropoles ne cesse de baisser depuis déjà un demi-siècle. Il est désormais très inférieur à la croissance nationale. La méthodologie utilisée consiste à reconstruire par le bas (bottom up) l'ensemble de la hiérarchie urbaine du pays à partir d'une définition strictement morphologique des agglomérations urbaines. Cette approche permet de vérifier qu'il existe effectivement un «attracteur » de taille démographique qui se situe actuellement autour de 500000 habitants. Elle met aussi en valeur le rôle des fonctions administratives et politiques territoriales, notamment de capitale d'État.

\section{INDEX}

Keywords: metropolis, urban agglomeration, urban hierarchy, demographic statistic

Mots-clés: métropole, agglomération urbaine, hiérarchie urbaine, statistique démographique 


\section{AUTHORS}

\section{CATHY CHATEL}

Cathy Chatel, chatelcathy@yahoo.fr, est Post-doctorante, FAPESP (Fundação de Amparo à Pesquisa do Estado de São Paulo), UNESP (Universidade Estadual Paulista "Júlio de Mesquita Filho") Câmpus Presidente Prudente. Elle a publié récemment :

- Casanova-Enault L., Chatel C., 2017. Une grille chorématique des dynamiques spatiales pour expliquer l'évolution des territoires. Mappemonde, nº 119, http://mappemonde.mgm.fr/119as3/ - Chatel C., Moriconi-Ebrard F., 2016. Éditorial. Numéro spécial (volume 1) « Aux frontières de l'urbain. Petites villes du monde. Ensemble flou et transitoire ou avenir d'une société urbaine?», Territoire en Mouvement, $\mathrm{n}^{\circ} 32$, https://tem.revues.org/3950

- Chatel C., Beltrão Sposito M. E., 2015. Forma e expansão urbana no Brasil: fatos e hipóteses. Primeiros resultados do banco de dados BRASIpolis. Cidades, vol. 12, $\mathrm{n}^{\circ}$ 21, http:// revista.fct.unesp.br/index.php/revistacidades/issue/view/285/showToc

\section{FRANÇOIS MORICONI-EBRARD}

François Moriconi-Ebrard, fme@i-geopolis.org, est Directeur de recherche CNRS, UMR ESPACE, Chaire franco-brésilienne de l'État de São Paulo (Consul a Général de France / UNESP. Il a publié récemment :

- Moriconi-Ebrard F., Perez J., 2017. Shanghai et Guangzhou sont les deux agglomérations urbaines les plus peuplées du monde. Confins [En ligne], 30 | 2017, mis en ligne le 10 avril 2017, consulté le 08 mai 2017, http://confins.revues.org/11729 ; DOI : 10.4000/confins.11729 - Moriconi-Ebrard F., 2016. Territoire et peuplement : l'exception française universelle. In Martin P. (coord.), La France : une exception? Paris, Ellipses, p. 13-60.

\section{MARIA ENCARNAÇÃO BELTRÃO SPOSITO}

Maria Encarnação Beltrão Sposito, mebsposito@gmail.com, est Professeur, UNESP (Universidade Estadual Paulista "Júlio de Mesquita Filho") Câmpus Presidente Prudente. Elle a publié récemment :

- Silva W. R., Sposito M. E. B (Org.), 2017. Perspectivas da Urbanização. Reestruturação urbana e das cidades (No Prelo, contrato assinado em 2016). Rio de Janeiro, Consequência, v. 1, 192 p.

- Sposito M. E. B., 2016. Oportunidades e desafios da pesquisa urbana comparada. In Firkowski, O. et al. (Org.), Estudos urbanos comparados: oportunidades e desafios da pesquisa urbana comparada. San Miguel de Tucumán, Universidad Nacional de Tucumán, p. 25-60.

- Sposito M. E. B., Maia D. S., 2016. Uma pesquisa em rede: cidades médias em análise. In Sposito M. E. B., Maia D. S. (Org.), Agentes econômicos e reestruturação urbana e regional: Dourados e Chapecó. São Paulo, Cultura Acadêmica, v. 1, p. 7-28. 